\title{
Influence of Electric Field on Recombination Dynamics of Quantum Confined Carriers
}

\author{
K.P. Korona ${ }^{a, *}$, J. Borysiuk ${ }^{b}$, C. Skierbiszewski ${ }^{c}$ \\ AND S. POROWSKI ${ }^{c}$ \\ ${ }^{a}$ Institute of Experimental Physics, Warsaw University \\ Hoża 69, 00-681 Warsaw, Poland \\ ${ }^{b}$ Institute of Electronic Materials Technology \\ Wólczyńska 133, 01-919 Warsaw, Poland \\ ${ }^{c}$ Institute of High Pressure Physics (UNIPRESS) \\ Sokołowska 31, 01-142 Warsaw, Poland
}

\begin{abstract}
We present time-resolved photoluminescence measurements of $\mathrm{GaN} / \mathrm{AlGaN}$ low dimensional structures showing very characteristic changes of dynamics related to strong electric field. Strong piezoelectric and spontaneous polarizations built-in in nitride structures lead to the changes in spatial separation of carriers which leads to changes in recombination energies and radiative lifetimes of the carriers. The observed effect can be well described by a simple exponential relation. The observed dependence can be explained by an approximated model of quantum-confined Stark effect based on the Airy functions.
\end{abstract}

PACS numbers: 78.47.+p, 72.20.Jv, 78.55.Cr

\section{Introduction}

GaN/AlGaN structures exhibit many interesting properties that are related to internal electric fields caused by spontaneous and piezoelectric polarizations. These polarizations result from differences in $\mathrm{Al}$ content in neighboring layers [1]. The field can be used to attract electrons which leads to the formation of 2-dimensional electron gas and gives a possibility of polarization doping. The AlGaN/GaN structures are used for production of ultraviolet detectors, light emitting diodes and lasers [2-4].

*corresponding author; e-mail: kkorona@fuw.edu.pl 
Here we present time-resolved photoluminescence (TRPL) measurements of $\mathrm{GaN} / \mathrm{AlGaN}$ low dimensional structures showing very characteristic changes of dynamics related to strong electric field. Piezoelectric and spontaneous polarizations built-in in nitride structures cause changes in the spatial separation of carriers which leads to the changes in recombination energies and radiative lifetimes of the carriers. The effect is well known $[5,6]$, however in our sample it exhibits a very clear exponential dependence, which gives an opportunity for a deeper analysis.

\section{Experimental details}

The investigated structure consisted of three GaN quantum wells (QWs) of the width: $2 \mathrm{~nm}$ (QW1), $4 \mathrm{~nm}$ (QW2), and $6 \mathrm{~nm}$ (QW3) embedded in $\mathrm{Al}_{0.15} \mathrm{Ga}_{0.85} \mathrm{~N}$ barriers. It was grown by $\mathrm{MBE}$ technique on bulk, slightly misoriented GaN substrates.

Transmission electron microscope images showed a good quality of the structures. Dislocations with $\boldsymbol{b}=\frac{1}{3}[11 \overline{2} 0]$ well-defined over full layer width were observed.

TRPL was measured from 10 to $300 \mathrm{~K}$. Frequency-doubled pulses (150 fs) of a Ti:sapphire laser had been used for photoexcitation of the sample. A monochromator and a streak camera gave the spectral and temporal distribution of the PL.

\section{Results and discussion}

At $T=8 \mathrm{~K}$, the sample showed PL from excitons in the GaN barrier $\left(\mathrm{X}_{\mathrm{GaN}}\right)$ at $3.47 \mathrm{eV}$ and had three PL bands from quantum wells: QW1 at 3.51, QW2 at 3.37, and QW3 at $3.21 \mathrm{eV}$ (see Fig. 1). The decay rates were $2.5 \mathrm{~ns}^{-1}, 0.12 \mathrm{~ns}^{-1}$ and $0.02 \mathrm{~ns}^{-1}$ for QW1, QW2, and QW3, respectively. Moreover, an additional band (QS) consisting of a few narrow lines (Q1, Q2 ..) was present at energies $3.40-3.43 \mathrm{eV}$.

The QS band was unusual (see Fig. 2). Most probably its different peaks came from the $4 \mathrm{~nm}$ QW (QW2), from places with different thicknesses or different electric field. The step-like changes of electric field could be due to partial relaxation of strain. The peaks were relatively narrow which suggested that the QW was of a very good quality between the steps. Decay rates of luminescence were from 1 to $6 \mathrm{~ns}^{-1}$ and changed strongly from peak to peak. As can be observed in Fig. 2B the dependence of decay rate versus energy was practically exponential. Moreover, the main peak of QW2 was in line with the observed changes between the QS peaks, which confirmed that QS originated from QW2. A similar but very weak band was observed above QW3.

TRPL measurements in various temperatures showed that energies of the PL peaks had the so-called S-shape dependence. When temperature increased the energy initially increased (which could be explained by delocalization of carriers) then (at $T>150 \mathrm{~K}$ ) energy decreased which was due to the gap-shrinkage effects $[7,8]$. The analysis of the QW1's and QW2's decay times showed that their temperature dependences were different. At low-temperature range, the delocal- 


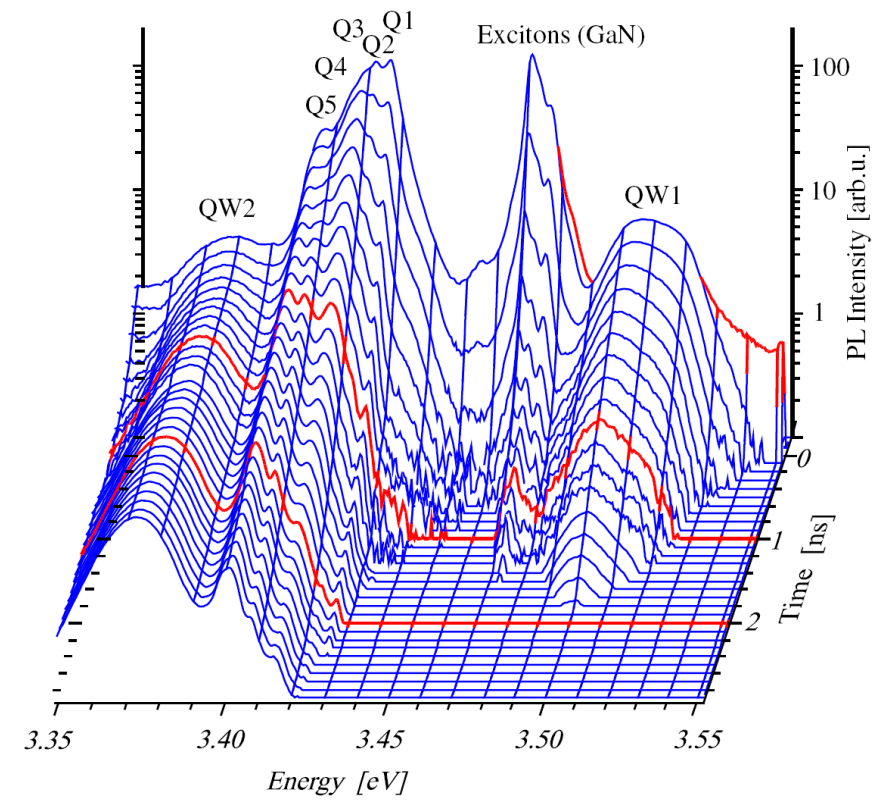

Fig. 1. Time-resolved spectrum of the sample with GaN/AlGaN quantum wells at $10 \mathrm{~K}$.

ization of carriers inside QWs led to an increase in lifetimes in QW1, whereas in QW2 delocalization caused a decrease in lifetimes (faster decay), which could be explained by the influence of electric field [7]. Activation energies for this process were $8 \mathrm{meV}$ and $16 \mathrm{meV}$, for QW1 and QW2, respectively. A thermally activated escape from QWs started above $150 \mathrm{~K}$ with activation energies of $0.13 \mathrm{eV}$ and $0.3 \mathrm{eV}$, for QW1 and QW2, respectively.

For the QS band every peak had a different temperature dependence. In average, the thermal escape dependence was similar to QW2, however, the delocalization of the dependence was similar to QW1.

In general, the delocalization and thermal escape rates depend exponentially on energy [9]. Therefore, one can try to explain the observed exponential lifetime vs. energy dependence, basing on these processes. However, the thermal escape is negligible in low temperature and the dependence of delocalization seemed to be too slow to explain the effect.

In another way, the effect of exponential dependence of QS lifetimes vs. energy can be explained by the quantum-confined Stark effect. When electron is confined in QW with high barriers (like in GaN/AlGaN case) it cannot drift in electric field $F$. Instead, its wave function $\psi(z)$ is squeezed. The Schrödinger equation should be completed with potential $V=-e F z$. In the case of rectangular QW with infinite barriers the solution are the Airy functions [10]:

$$
\psi(z)=a^{*} \operatorname{Ai}((e F z-E) / U)+b^{*} \operatorname{Bi}((e F z-E) / U),
$$



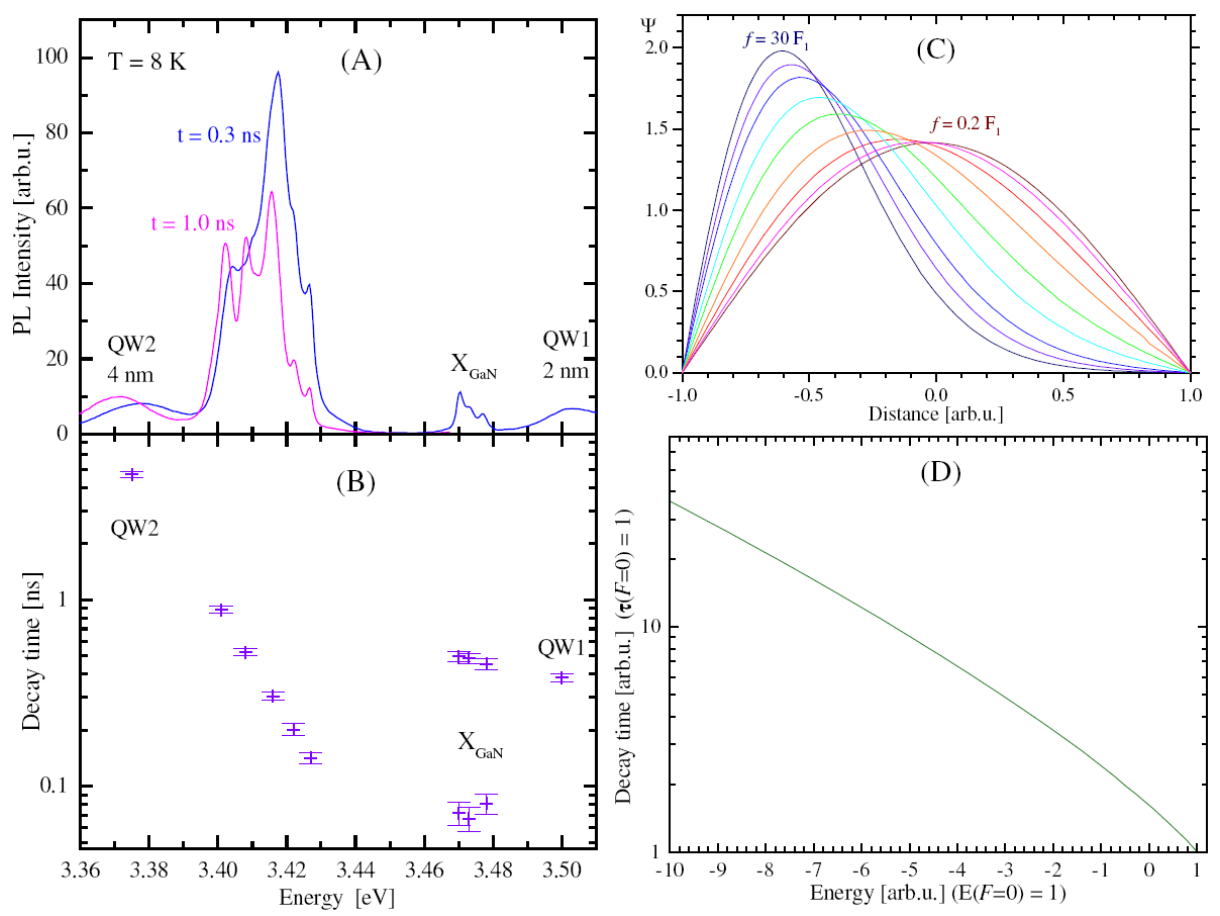

Fig. 2. (A) PL spectra of the sample measured at $0.3 \mathrm{~ns}$ and $1.0 \mathrm{~ns}$ after excitation. (B) Decay times of the peaks seen above. (C) Wave functions of electron in QW in electric field. (D) Calculated recombination times vs. energy.

where $E$ is the energy eigenvalue and $U=2\left(\pi^{2} m^{*}\right)^{1 / 3} /(e h F)^{2 / 3}$. The functions are plotted in Fig. 2C.

The recombination rate is proportional to the overlap of electron and hole. It is not possible to strictly calculate it in an analytical way, so recombination times were calculated numerically and the results are plotted in Fig. 2D. It has been found that $\tau(E)$ dependence is nearly exponential.

This result can be understood in the following way. For high electric field, the Airy function parameter $e F z-E$ is positive in a part of the QW. Since Bi function increases very fast for positive parameters, borders conditions implies that $b \approx 0$. On the other hand, Ai function for positive parameters exponentially decreases. The electron function has a maximum near high potential border and decreases exponentially in negative-border direction. The shape of hole wave function is similar but reversed. So integral of the overlap should be an exponent. In fact, it can be approximated by

$$
M_{\mathrm{eh}}=C \exp \left(-\left(2\left(E_{\mathrm{e}} U_{\mathrm{h}}-E_{\mathrm{h}} U_{\mathrm{e}}+E_{\mathrm{g}}\right) / 3 U_{\mathrm{e}} U_{\mathrm{h}}\right)^{2 / 3}\right) .
$$

Since decay time $\tau \sim 1 / M_{\mathrm{eh}}^{2}$, so this explains nearly exponential dependence of $\tau$ vs. energy. 
It can be noticed also that the energy shift due to electric fields depends only on the product of field and thickness, $F L$. Therefore, we cannot distinguish if the steps in energy were due to steps of the QW width $L$ or due to the electric field $F$. However, the steps are so fine that they must be caused by smaller than monolayer steps in the QW width. Taking this into account, the energy steps are most probably due to strain relaxation steps causing gradual changes of the piezoelectric effect.

\section{Conclusions}

Time resolved photoluminescence was measured on the misoriented GaN/AlGaN low dimensional structure showing a set of sharp PL lines at energies slightly above QW's energy. These lines are most probably due to relaxation steps causing gradual changes of the piezoelectric effect.

It was observed that the decay time of the sharp PL lines depended exponentially on energy of these lines. The observed dependence can be explained by an approximated model of the quantum-confined Stark effect based on the Airy functions, however also some transport processes should be included in the careful analysis of the dynamics.

\section{Acknowledgments}

This work was partially supported by grant No. 3 T11B 05430 (financed by Polish budget for science in years 2006-2008).

\section{References}

[1] F. Bernardini, V. Fiorentini, D. Vanderbilt, Phys. Rev. B 56, R10024 (1997).

[2] S. Nakamura, M. Senoh, N. Iwasa, S.-I. Nagahama, Appl. Phys. Lett. 67, 1868 (1995).

[3] F. Omnès, E. Monroy, in: Nitride Semiconductors, Handbook on Materials and Devices, Eds. P. Ruterana, M. Albrecht, J. Neugebauer, Wiley-VCH Verlag, Berlin 2003, p. 664.

[4] K.P. Korona, A. Drabińska, K. Pakuła, J.M. Baranowski, Acta Phys. Pol. A 110, 211 (2006).

[5] T. Takeuchi, S. Sota, M. Katsuragawa, M. Komori, H. Takeuchi, H. Amano, I. Akasaki, Jpn. J. Appl. Phys. 36, L382 (1997).

[6] Jin Seo Im, H. Kollmer, J. Off, A. Sohmer, F. Scholz, A. Hangleiter, Phys. Rev. B 57, R9435 (1998).

[7] P. Lefebvre, A. Morel, M. Gallart, T. Taliercio, J. Allègre, B. Gil, H. Mathieu, B. Damilano, N. Grandjean, J. Massies, Appl. Phys. Lett. 78, 1252 (2001).

[8] B. Chwalisz, A. Wysmołek, R. Bożek, K.P. Korona, R. Stępniewski, W. Knap, K. Pakuła, J.M. Baranowski, N. Grandjean, J. Massies, P. Prystawko, I. Grzegory, Acta Phys. Pol. A 103, 573 (2003).

[9] K.P. Korona, P. Wojnar, J.A. Gaj, G. Karczewski, J. Kossut, J. Kuhl, Solid State Commun. 133, 369 (2005).

[10] D.A.B. Miller, D.S. Chemla, T.C. Damen, A.C. Gossard, W. Wiegmann, T.H. Wood, C.A. Burrus, Phys. Rev. B 32, 1043 (1985). 